\title{
Sleep problems from infancy are linked with impaired well-being in middle childhood
}

\author{
By Dr. Jessica Edwards
}

Researchers in the USA and Australia have found that sleep disturbances from early childhood are associated with reductions in well-being at age 10-11 years old. Ariel Williamson and colleagues came to this conclusion after analysing data from $>5,000$ children enrolled in the Longitudinal Study of Australian Children - Birth Cohort. They evaluated caregiver reports on child sleep problems every two years from birth to age 10-11 years, and the outcomes of caregiver-reported, teacher-reported and child-completed tasks that indicated a child's well-being at age 10-11 years.

Williamson et al. found five distinct sleep problem trajectories: persistent problems from infancy through to middle childhood ( $7.7 \%$ of the sample), limited infant / preschool sleep problems ( $9.0 \%$ ), increased middle childhood sleep problems (17.0\%), mild sleep problems over time (14.4\%) and no sleep problems (51.9\%). Compared to children with no sleep problems, they found that those with persistent problems had impairments in emotional and behavioural functioning, health related quality of life and academic skills, but not perceptual reasoning. Children with other sleep problem trajectories also had impairments in some domains compared to those with no sleep problems.

In a press release published by the Children's Hospital of Philadelphia, Williamson said "For both teacher-rated and parent-rated mental health concerns, such as internalising and externalising problems, children with persistent sleep problems had the greatest impairments. But we also found that even kids with sleep problems limited to early childhood or mild sleep problems over time had more emotional and behavioural concerns compared to the children with no sleep problems". Williamson went on to urge caution, explaining that "just because [a] child has a sleep problem, it doesn't mean they are destined for mental health concerns later in life."

The researchers suggest that any parent who is concerned about their children's sleep should consult their paediatrician and consider seeking sleep treatment. Similarly, they believe that screening for child sleep problems at every well child visit is important to address sleep concerns. Going forward, they consider that studies which investigate whether early interventions targeting sleep problems could prevent later impaired child well-being are now warranted.

Referring to:

Williamson, A.A., Mindell, J.A., Hiscock, H. \& Quach, J. (2020), Longitudinal sleep problem trajectories are associated with multiple impairments in child well-being.

J. Child Psychol.

Psychiatr. doi: 10.1111/ jcpp.13303.

\section{Glossary:}

\section{Internalising} problems: emotional symptoms which tend to be expressed internally, such as anxiety and low mood.

Externalising
problems:
maladaptive
behaviours which are
directed externally
towards the
environment, such as
aggressive behaviours
and impulsivity.

